\title{
How language proficiency contributes to Chinese students' academic success in Korean universities
}

\author{
Wei Yan ${ }^{*}$ and Liying Cheng ${ }^{*}$
}

\author{
* Correspondence: \\ yan.wei@queensu.ca; \\ liying.cheng@queensu.ca \\ Faculty of Education, Queen's \\ University, Kingston, ON, Canada
}

\begin{abstract}
The present study investigated the key determinants of Chinese students' academic success in terms of GPA and the number of credit hours earned in Korean Universities. The determinants investigated included gender, age, prior academic performance, academic self-efficacy, the TOPIK score, self-perceived Korean and English proficiency, and the previous length of Korean and English study. This study specifically focused on three research questions concerning the prediction of Chinese students' academic success in Korean universities, the additional contribution of Korean and English language proficiency, and the examination of prediction patterns for undergraduate and graduate students.

A questionnaire was issued and collected from 138 undergraduate and 63 graduate Chinese students studying in 27 different Korean universities. The questionnaire consisted of four sections: demographic information, academic background, language proficiency and psychological factors. Correlation and multiple regression analyses were conducted to address the proposed research questions.

The findings demonstrated that traditional factors, including gender and prior academic performance, were effective predictors of academic success. However, academic self-efficacy did not play an influential role in participants' academic success. Language proficiency had a moderate effect on Chinese students' academic success, which is consistent with previous studies that reported a positive statistically significant relationship between language proficiency and academic success. The different natures of undergraduate and graduate studies determined that the predictors of undergraduate and graduate students' academic success were different.

The present study addressed the research gap by integrating theoretical constructs from both psychology and language education, and also by exploring the relationships between language proficiency and academic success in a less researched test, TOPIK, and in two second languages, Korean and English, at the same time. The findings of this study contribute to the overall understanding of international students' academic success, in particular the success of Chinese students studying in Korean universities.

Keywords: Test of Proficiency in Korean (TOPIK); Language proficiency; Academic success; Academic self-efficacy; Chinese students
\end{abstract}

Current research on international students has focused on the relationship between language proficiency and academic success. Academic success is typically measured by Grade Point Average (GPA) whereas language proficiency is most often evaluated using international tests such as the Test of English as a Foreign Language (TOEFL) and the International English Language Testing System (IELTS). As more and more

(c) 2015 Yan and Cheng. This is an Open Access article distributed under the terms of the Creative Commons Attribution License (http://creativecommons.org/licenses/by/4.0), which permits unrestricted use, distribution, and reproduction in any medium, provided the original work is properly credited. 
international students are travelling to English speaking countries for higher education, it has become essential that they attain a sufficient level of English language proficiency since academic lectures, assignments, and communications are all conducted in English. In fact, cut-off scores on TOEFL and IELTS are set by each university for admission purposes.

It is evident that the setting of such cut-off scores rests upon the widely accepted assumption that a certain level of language proficiency is necessary for academic success (Cotton \& Conrow, 1998; Kerstjens \& Nery, 2000). However, the relationship between language proficiency and international students' academic success is complex and difficult to demonstrate, and the results from previous studies are mixed and highly inconclusive (Cho \& Bridgeman, 2012). This study addresses this relationship in a unique context, investigating how language proficiency contributes to Chinese students' academic success in South Korean universities.

Chinese students' enrolment in Korean universities has been increasing steadily since China and South Korea established formal diplomatic relations in 1992 (Gao, 2010). Currently, there are more than 80,000 Chinese students studying in over 400 South Korean universities (Gukrip Gukje Gyoyukwon [National Institute for International Education], 2013; Shin, 2011). When applying to universities in Korea, international students need to prove their Korean language proficiency since they will be using Korean as the medium of instruction once admitted. In order to gain admission, they are required to submit their scores on the Test of Proficiency in Korean (TOPIK). The TOPIK is a test of Korean language proficiency and consists of three levels: TOPIK Elementary, TOPIK Intermediate, and TOPIK Advanced. The increasing number of Chinese students taking TOPIK and choosing to study in Korea indicates a necessity for a study on this group of students to understand what contributes to their academic success.

As a language proficiency test, TOPIK functions the same way as TOEFL and IELTS for those who pursue higher education in South Korea. Whereas prediction studies on TOEFL and IELTS have been performed frequently in language testing, research on the predictive validity of TOPIK has been scarce. The inconsistent results from previous prediction studies also point out that further empirical investigation into the relationship between language proficiency and academic success is needed, especially for a less commonly researched test such as TOPIK.

Passing scores for TOPIK Intermediate are required by most admission offices in Korean universities. However, there appears to be no empirical rationale supporting this cut-off score decision. Furthermore, while TOPIK scores have been widely accepted as an admission benchmark among Korean universities, it is unclear whether TOPIK measures the Korean language proficiency required in academic settings.

The determinants of academic success in higher education have been researched in both education and psychology for decades. However, there has been little integration of this research between the two disciplines (Robbins et al., 2004). To address this gap, Robbins et al. (2004) brought the two bodies of literature together and used a metaanalysis to increase understanding. They reported that the best predictors for GPA are academic self-efficacy and achievement motivation. The inclusion of these psychological elements improved predictive accuracy above the traditional predictors of demographics and prior academic performance. The integration of these factors is therefore expected to enhance our overall comprehension, and thus the present study includes 
both traditional and psychological factors to explore the academic success of Chinese students studying in Korean universities.

\section{Research context}

The TOPIK was developed by the Korea Research Foundation in 1997 and is currently administered by the National Institute for International Education (NIIED) up to four times per year in Korea and twice yearly in 53 other countries (Gukrip Gukje Gyoyukwon [National Institute for International Education], 2010). By 2012, a total of 28 tests had been administered, and more than one million test-takers had written the test (Yu, 2013). Among those who took the twenty-ninth TOPIK in January 2013, $44.5 \%$ intended to seek higher education in Korea (Yu, 2013).

Four areas - Vocabulary \& Grammar, Writing, Listening, and Reading - are tested in all three levels of TOPIK, with 100 points for each area and a maximum score of 400. All test questions are multiple choice with the exception of the Writing subsection, which encompasses sentence completion, paragraph completion and a short essay, in addition to the multiple choice questions (Gukrip Gukje Gyoyukwon [National Institute for International Education], 2010). Under each level of TOPIK, there are two sublevels depending on the scores of the applicants. In total there are six sublevels: Levels 1 and 2 (TOPIK Elementary), Levels 3 and 4 (TOPIK Intermediate), and Levels 5 and 6 (TOPIK Advanced). The Korean Ministry of Education requires all international students to achieve at least Level 3 in order to study in Korean universities, while a large number of universities require Level 4 for admission (Yu, 2013). In July 2014, the NIIED released the newly restructured TOPIK: TOPIK I and TOPIK II. Based on the levels of difficulty, TOPIK I is equal to the old TOPIK Elementary, and TOPIK II is equal to TOPIK Intermediate and Advanced.

In South Korean universities, English language proficiency is an additional asset to international students. Students with a higher level of English proficiency have a better chance of being accepted by prestigious universities and achieving academic excellence. For example, English is a required subject in Korean universities' entrance exams; the higher the score earned on the English portion of the exam, the greater the opportunities that student will have entering a prestigious university. English is also an advantage for international applicants to Korean universities, especially to graduate schools, as students with certain levels of TOEFL or IELTS scores are granted more scholarships than those who only submit TOPIK scores.

Students with a high level of English are favoured because of the increased use of English as a medium of instruction in Korean universities. Even in Korean-medium courses, English textbooks are often adopted, especially at the graduate level. Thus the academic success of Chinese students studying in Korean universities may be impacted not only by their proficiency in Korean as a second language, but also by their proficiency in English as a third language. The present study hence contributes to the understanding of the relationships between language proficiency and academic success by exploring both Korean and English language proficiency at the same time.

\section{Relationship between language proficiency and academic success}

Graham (1987) reviewed 19 early predictive validity studies and attempted to analyze the relationship between various English proficiency test scores (mostly TOEFL) and 
academic success. She grouped the studies according to the conclusions drawn by the researchers, which were negative conclusions, mixed conclusions, and positive conclusions. The results of prediction studies conducted after Graham (1987) remain inconsistent. A number of them found significant correlations between TOEFL scores and GPAs (e.g., Johnson, 1988; Light \& Teh-Yuan, 1991; Stoynoff, 1997; Wait \& Gressel, 2009), while others concluded that TOEFL was not a significant predictor of academic success (e.g., Ayers \& Quattlebaum, 1992; Krausz et al., 2005; Nelson et al., 2004).

More recently, Cho and Bridgeman (2012) examined the relationship between the newly introduced TOEFL iBT scores and GPA of both undergraduate and graduate international students in the United States. A total of 2594 students, including 744 undergraduate and 1850 graduate students, were represented in the data. The results showed that TOEFL iBT accounted for approximately $4 \%$ of the variance in GPA for graduate students $(r=.20)$ and $3 \%$ for undergraduate students $(r=.18)$. It is noteworthy that even with such a large sample group, the statistically significant correlations were rather small.

Similarly, the results of studies investigating the predictive validity of IELTS were also inconsistent. Some studies indicated a significant correlation between IELTS and academic success (e.g., Elder, 1993; Feast, 2002; Hill et al., 1999; Woodrow, 2006), while others found no correlation or only subsection score correlations with GPA (e.g., Cotton \& Conrow, 1998; Dooey \& Oliver, 2002; Kerstjens \& Nery, 2000). These mixed findings suggest that the relationship between language proficiency and academic success is complicated, and is not a one-to-one correspondence (Cho \& Bridgeman, 2012). Regardless of the size of sample group, the level of study, or the type of language proficiency test used, the results have been mixed and inconclusive.

When statistically significant correlations were found, they were relatively small. Criper and Davies (1998) suggested that one should not expect a correlation of more than 3 (about $10 \%$ ) in this type of research. This is partly due to an inherent design problem of predictive validity studies. Specifically, only students who have been admitted to the university are included in the data, which means the datasets consist of restricted samples comprising only those whose TOEFL or IELTS scores achieved a certain level (above the cut-off score). These truncated samples do not represent a full range of scores. Therefore, the correlations obtained would be lower than the actual correlations.

University students' academic performance in general has also been intensively investigated. A comprehensive meta-analysis conducted by Richardson, Abraham, and Bond (2012) systematically reviewed 7167 articles published over 13 years (19972010) of research on the prediction of university students' GPAs. Correlations showed that older students and female students obtained higher grades in universities. For the traditional correlates of GPA, high school GPA, SAT, and ACT were found to have medium-sized correlations with undergraduate GPA as expected. Among 42 psychological factors, a large correlation was observed for performance self-efficacy, and medium-sized correlations with GPA were shown for grade goal, effort regulation, and academic self-efficacy. Richardson et al. (2012) therefore concluded that a combination of motivation (performance self-efficacy, grade goal, and academic self-efficacy) and self-regulatory capacity (effort regulation) best predicted university students' GPAs.

Although the four key psychological factors identified by Richardson et al. (2012) have potential importance for the present study, performance self-efficacy and grade 
goal are expectations of future academic success. As such, these factors were not suitable for inclusion because participants already knew their performance by the time they engaged in the current study. Therefore, only academic self-efficacy and effort regulation were incorporated here. Academic self-efficacy refers to students' anticipation of their performance on the basis of generalized perceptions of academic capability. Effort regulation includes self-management of motivation or persistence when challenged by difficult work. Neither of these factors has been researched on Chinese students studying abroad.

Although Richardson et al.'s (2012) study did not specifically target the university GPAs of international students, it also did not exclude this group. For example, Stoynoff's (1997) study of non-native English speaking freshmen at one American university was included in the meta-analysis. Moreover, university admission requirements for local students and international students are very similar, except for the fact that international students need, additionally, to provide proof of English proficiency. This prerequisite suggests that language proficiency is the only important difference for international students. Therefore, the results of Richardson et al.s (2012) study can likely be applied to international students including the subjects of the present study.

\section{Indicators of language proficiency and academic success}

Though most previous prediction studies have used scores from commercial tests such as TOEFL or IELTS to measure English language proficiency, some universities conducted their own English proficiency tests to place incoming international students. There are studies that used these institutional tests to measure language proficiency (Lee \& Greene, 2007; Light \& Teh-Yuan, 1991), but it may not always be practical to administer such tests to all potential applicants and hence institutional proficiency tests are not feasible for university admission purposes.

Apart from the institutional proficiency tests mentioned above, previous studies have also included other indicators of language proficiency such as length of previous English language study (e.g., Wimberley, McCloud, \& Flinn, 1992; Woodrow, 2006; Xu, 1991), on-campus English program support (e.g., Cotton \& Conrow, 1998; Hill et al., 1999; Wait \& Gressel, 2009), and self-perceived English language proficiency (e.g., Li, Chen, \& Duanmu, 2010; Light \& Teh-Yuan, 1991; Xu, 1991). In Xu's (1991) study, selfrated English proficiency, though not a traditional predictive validity study of academic success, was the most significant predictor of the perceived level of academic difficulty while the TOEFL score was not found to be significant. These findings suggest that along with scores on commercial language proficiency tests, other measures of language proficiency may be useful for predictive purposes. To this end, the present study includes the length of previous language study and self-perceived language proficiency, as well as scores on standardized tests such as the TOPIK.

Studies on university students' academic success and on predicative validity have largely explored the various factors, including language proficiency, that affect GPA. However, Graham (1987) questioned whether GPA was always a valid indicator of academic performance. Heil and Aleamoni (1974) pointed out that GPA did not take into account the number of courses taken. For example, an international student with poor English proficiency may only take two courses and yet still maintain a high GPA. In response to Graham (1987), several researchers also used the number of credits earned as 
an indicator of academic success. For example, Light, $\mathrm{Xu}$, and Mossop (1987) found that the correlation between TOEFL scores and graduate credits earned was significant. It was also slightly higher than the correlation between TOEFL scores and GPA in the same study (also see Johnson, 1988; Stoynoff, 1997). Nevertheless, GPA remains the most commonly used indicator for academic success in prediction studies. It is still the key criterion for postgraduate selection and graduation employment, and is predictive of occupational status (Strenze, 2007). Thus, this study investigated two prevailing indicators of academic success: GPA and credit hours earned.

\section{Research questions}

To summarize, traditional factors including gender, age, and prior academic performance (high school GPA for undergraduate students and undergraduate GPA for graduate students) were included in the present study. Furthermore, guided by Robbins et al. (2004), psychological factors such as academic self-efficacy and effort regulation were integrated with these traditional educational factors in order to predict Chinese students' academic success in Korean universities. The integration of theoretical constructs from both education and psychology was considered essential for obtaining an overall understanding of university students' academic success (Robbins et al., 2004). Looking at language proficiency alone is inconclusive and so combining test scores with selfperceived proficiency and the length of previous language study was necessary to provide a clearer picture. Since English plays a crucial role in academic success in Korean universities, both Korean and English language proficiencies were examined in this study using the factors mentioned above.

The indicators of academic success included both GPA and the number of credit hours earned, especially in the first semester, given that the relationship between language proficiency and academic success was the strongest at this point in time (Elder, 1993; Light et al., 1987). The first semester GPA is the most frequently used measure for judging academic success in predictive validity studies (Kerstjens \& Nery, 2000). In fact, the first semester GPA can be a strong predictor of overall GPA (Nelson et al., 2004). Hence, both GPA and credit hours from the first semester were included in the present study.

This study investigated the key determinants of Chinese students' academic success in terms of GPA and the number of credit hours earned in Korean universities. The determinants investigated included gender, age, prior academic performance, academic self-efficacy, effort regulation, and especially Korean and English language proficiency including TOPIK scores, self-perceived Korean and English proficiency, and the length of previous Korean and English study. Note that standardized English proficiency test scores were not included in this study because only few Chinese students who study in Korean universities take English proficiency tests such as TOEFL or IELTS. Specifically, the following research questions were addressed:

1. What are the predictors of Chinese students' academic success in Korean universities in terms of GPA and the number of credit hours earned?

2. What do Korean and English language proficiency indicators add to the contribution of traditional and psychological predictors of academic success?

3. Are the prediction models similar for undergraduate and graduate students? 


\section{Method \\ Participants}

The participants of this study were 138 undergraduate and 63 graduate Chinese students attending 27 universities throughout South Korea. They were full-time, degreeseeking students who took TOPIK Intermediate and started their current studies in the years 2011 and 2012. TOPIK refers to TOPIK Intermediate hereafter. This study only included master's students as graduate participants. Among 138 undergraduate participants, there were 72 male $(52.2 \%)$ and 66 female $(47.8 \%)$ students. The ages of undergraduate participants varied from 17 to 28 years, with a mean of 23.38. Among 63 graduate participants, there were 16 male (25.4 \%) and 47 female (74.6\%) students. Graduate participants' ages ranged from 21 to 33 years, with a mean of 25.98. As mentioned earlier, participants were from 27 different universities. Fifteen of those universities were located in Seoul, and provided 79 undergraduate $(57.2 \%)$ and 49 graduate (77.8 \%) participants.

\section{Instrument}

Data were obtained through a questionnaire, which comprised four sections: demographic information (e.g., gender, age), academic background (e.g., prior academic performance, GPA and credit hours earned in the first semester), language proficiency (e.g., TOPIK score, self-perceived Korean and English proficiency, the length of previous Korean and English study) and psychological factors (e.g., academic self-efficacy, effort regulation). Richardson et al. (2012) identified and mapped both academic self-efficacy and effort regulation in the Motivated Strategies for Learning Questionnaire (MSLQ) (Pintrich et al., 1991). The present study adopted the MSLQ for the fourth part of the questionnaire to measure participants' academic self-efficacy and effort regulation.

The MSLQ, developed by Paul Pintrich and his colleagues, is a well-established and widely used instrument for assessing university students' motivation and learning strategies with the ultimate goal of helping students improve learning. Its current form was finalized in 1991, and both academic self-efficacy and effort regulation were mapped into Pintrich's (2004) recent conceptual framework for self-regulated learning in college students. The MSLQ has been used in different languages, in different countries, and on diverse samples and settings (see Duncan \& McKeachie, 2005). It is completely modular, and the subscales can be used together or individually, depending on the needs of the researcher or instructor (Pintrich et al., 1991).

The questionnaire was designed in English but was administered in Chinese to ensure that there was no language difficulty in its completion. The first three parts were translated into Chinese, and then back translated to ensure accuracy. For the fourth part, Wu and Cheng's (1992) Chinese version of the MSLQ was adopted.

\section{Data collection}

Questionnaires were administered and collected via both paper and web-based versions, and participants were recruited on a voluntary basis. Colleagues and friends in five Korean universities helped us gain access to Chinese students through international centers, Korean culture classes, and Chinese students' associations. The first author introduced the study, invited potential participants and issued the paper-based questionnaire to them. In addition, participants were asked to distribute cards with a link to the 
web-based version of the questionnaire to their friends on a voluntary basis, inviting them to also participate the study. The web-based questionnaire was additionally spread via online forums, social networking service groups, and online chat groups where Chinese students who study in Korea are actively involved.

\section{Data analyses}

SPSS (Version 19.0) was used to analyze the data in order to answer the research questions. Descriptive statistics were performed first, and missing data were carefully examined. Gender was recoded: male as 1, and female as 2. Unfortunately, only 102 undergraduate and 51 graduate participants reported their TOPIK scores, which reduced the sample size for correlation and regression analyses, and limited the present study. The comparison of descriptive statistics showed that participants who finished the paper-based questionnaires closely approximated those who finished the web-based questionnaires in terms of key variables. Therefore, both paper and web-based versions of the questionnaire were analyzed together.

Factor analysis with principal component analysis and direct oblimin rotation was then conducted on the items of self-perceived language proficiency and psychological factors. Principal component analysis was the most common factor extraction technique in the language assessment literature (Ockey, 2014). Direct oblimin rotation was used because it allowed for the factors to be correlated (Fabrigar et al., 1999). Factor analyses were first conducted with undergraduate and graduate participants separately. The results were alike, which showed that the underlying constructs for both groups of participants were the same. Therefore, factor analyses were repeated combining both undergraduate and graduate participants.

Items involving Korean language proficiency (overall, reading, writing, listening and speaking ratings) were neatly loaded together, as were the items of English language proficiency (overall, reading, writing, listening and speaking ratings). Therefore, these two factors were named as self-perceived Korean proficiency and self-perceived English proficiency. Self-perceived English proficiency was extracted as the first factor, which accounted for $49.02 \%$ of the total variance. Self-perceived Korean language proficiency was the second factor extracted, and accounted for an additional $31.06 \%$ of the total variance. The Cronbach's alpha for items loading on self-perceived English language proficiency was 0.96 , and 0.91 for self-perceived Korean language proficiency.

Items of academic self-efficacy loaded together, representing the same construct in the original design of the MSLQ. It accounted for $51.68 \%$ of the total variance. The Cronbach's Alpha of items loading on academic self-efficacy was .94. As for effort regulation, the two negatively worded items loaded together while the two positively worded items also loaded together. These accounted respectively for $13.12 \%$ and $9.32 \%$ of the total variance in the psychological constructs. The four items of effort regulation did not load together as one factor. Therefore, these four items were discarded, and only the academic self-efficacy factor was used during further analyses. Note that Rao and Sachs (1999) have already recognized that Chinese respondents may have trouble answering reverse-coded items. The present study confirmed that Chinese students indeed have such difficulties (see also Rao et al., 2000; Sachs et al., 2001).

Correlation analyses were completed with the dependent variables (first semester GPA and credit hours) and all independent variables including gender, age, prior 
academic performance (high school GPA for undergraduate participants and undergraduate GPA for graduate participants), the length of previous Korean and English study, the TOPIK total score, self-perceived Korean and English proficiency, and academic self-efficacy. Correlation analyses were then performed among the independent variables mentioned above to ensure that no highly correlated variables were entered in the regression at the same time.

Regression analysis is one of the most commonly used methods in prediction studies of academic success (McKenzie \& Schweitzer, 2001; Li et al., 2010). Multiple regression was used to address the research questions of this study. For undergraduate participants, two regression models were conducted with first semester GPA and first semester credit hours earned used as the dependent variables. Gender, age, prior academic performance (high school GPA), and academic self-efficacy were entered into the regression first. Then the TOPIK total score, self-perceived Korean proficiency, selfperceived English proficiency, the length of previous Korean study, and the length of previous English study were added using stepwise regression. Stepwise regression was used during the second step to determine which, if any, of the language proficiency indicators significantly predicted first semester GPA or credit hours and their unique contribution. The regressions were done in a similar manner for graduate participants. In the final step, regression models were compared between undergraduate and graduate participants.

\section{Results}

\section{Correlation analyses}

Pearson product-moment coefficients between independent variables and dependent variables for undergraduate and graduate participants are presented in Table 1 . The sample included 102 undergraduate and 51 graduate students. TOPIK subsection scores were also included in the correlation analyses in order to explore the relationship between the sub-skills of Korean proficiency and academic success.

As shown in Table 1, undergraduate participants' first semester GPAs were significantly correlated with most independent variables. GPAs were not significantly correlated with age or the length of previous Korean study. The correlation coefficients were low to moderately low, perhaps due to the restricted range in the sample. The highest correlation coefficient was with self-perceived Korean proficiency $(r=.36)$, followed by gender $(r=.32)$ and the TOPIK total score $(r=.29)$. On the other hand, undergraduate participants' first semester credit hours were only significantly correlated with age, indicating that older students had a tendency to take more courses. Undergraduate participants' first semester GPAs and credit hours were negatively correlated with each other, suggesting that the more credit hours undergraduate students took, the lower their GPAs.

Table 1 also showed that graduate first semester GPAs were significantly correlated with undergraduate GPA, the length of previous Korean and English study, TOPIK Vocabulary \& Grammar, Writing, and total scores. TOPIK Listening and Reading scores were not significantly correlated with first semester GPAs, probably due to the ceiling effect. The correlation coefficients were low to moderately low, perhaps due to the restricted range in the sample. The highest correlation coefficient was with the TOPIK Vocabulary \& Grammar score $(r=.41)$, followed by the TOPIK total and Writing scores $(r=.40)$ and undergraduate GPA $(r=.39)$. 
Table 1 Correlations between independent variables and dependent variables

\begin{tabular}{|c|c|c|c|c|}
\hline & \multicolumn{2}{|l|}{$\begin{array}{l}\text { Undergraduate } \\
(n=102)\end{array}$} & \multicolumn{2}{|l|}{$\begin{array}{l}\text { Graduate } \\
(n=51)\end{array}$} \\
\hline & First semester GPA & $\begin{array}{l}\text { First semester } \\
\text { credit hours }\end{array}$ & First semester GPA & $\begin{array}{l}\text { First semester } \\
\text { credit hours }\end{array}$ \\
\hline First semester GPA & & $-.25^{*}$ & & $-.28^{*}$ \\
\hline Gender & $.32^{* *}$ & -.18 & .21 & -.00 \\
\hline Age & -.17 & $.22^{*}$ & .04 & -.10 \\
\hline High school GPA & $.23^{*}$ & -.03 & - & - \\
\hline Undergraduate GPA & - & - & $.39 * *$ & -.00 \\
\hline Length of previous Korean study & -.03 & .17 & $.29^{*}$ & .26 \\
\hline Length of previous English study & $.25^{*}$ & .10 & $.29^{*}$ & -.07 \\
\hline TOPIK Vocabulary \& Grammar & $.20^{*}$ & -.16 & $.41^{* *}$ & -.13 \\
\hline TOPIK Writing & $.27^{* *}$ & -.14 & $.40^{* *}$ & .01 \\
\hline TOPIK Listening & $.28^{* *}$ & -.05 & .07 & $-.30^{*}$ \\
\hline TOPIK Reading & $.21^{*}$ & -.14 & .25 & $-.39^{* *}$ \\
\hline TOPIK Total & $.29^{* *}$ & -.13 & $.40^{* *}$ & $-.29^{*}$ \\
\hline Self-perceived Korean proficiency & $.36^{* *}$ & .04 & .10 & -.21 \\
\hline Self-perceived English proficiency & $.21^{*}$ & .06 & .14 & -.16 \\
\hline Academic self-efficacy & $.26^{* *}$ & -.03 & .07 & -.02 \\
\hline
\end{tabular}

Note: ${ }^{* *} p<.01,{ }^{*} p<.05$

Graduate participants' first semester credit hours had significant negative correlations with TOPIK Listening, Reading, and total scores. International students with insufficient Korean proficiency were required to take Korean language courses along with their major courses. Therefore, graduate students with insufficient Korean proficiency took more credit hours than those with strong Korean proficiency, which explained the above negative correlations. In contrast, Light et al. (1987) found a positive correlation between TOEFL scores and graduate credit hours $(r=.19)$ with 376 international graduate students studying in the United States. This inconsistency might be due to the fact that previous studies did not include students who are required to take additional English language classes. And those whose English proficiency is strong can take more courses in their fields. Graduate participants' first semester GPAs and credit hours were also negatively correlated with each other.

The overall correlation coefficients were rather small, which was expected due to the range restriction of the sample. However, even a small correlation can indicate a meaningful relationship (Cho \& Bridgeman, 2012; Rosenthal \& Rubin, 1982; Sackett et al., 2008; Schrader, 1965). This should be kept in mind when interpreting the correlations between TOPIK scores and indicators of academic success obtained in the present study. The TOPIK total score explained $8.41 \%(r=.29)$ of the variance in undergraduate GPA and $16 \%(r=.40)$ of the variance in graduate GPA. As such, TOPIK showed stronger predictive validity than did English proficiency tests in previous studies.

Correlations among independent variables were also examined. For undergraduate students, these varied to a maximum correlation of 0.47 (age with the length of Korean study). Once again, the correlations were generally small to moderately small, suggesting that these variables could be independently used in regression analyses. 
Interestingly, undergraduate students' gender and age were significantly correlated $(r=-.30)$. This indicated that female undergraduate students were younger than their male counterparts, which is possibly because female students were developmentally ready to start their schooling earlier. The correlations for graduate students varied to a maximum correlation of 0.52 (self-perceived Korean and English proficiency). The correlations were small to medium, suggesting that these variables could be independently used in subsequent analyses.

\section{Regression analyses}

Hierarchical regression analyses were conducted with first semester GPA and then first semester credit hours as the dependent variables. One hundred and two undergraduate and fifty-one graduate participants who reported their TOPIK total scores were included in the regressions respectively. The results of these analyses are shown in Tables 2, 3, 4, 5, and 6 .

Table 2 shows the results of multiple regression with the undergraduate participants' first semester GPAs as the dependent variable. Gender, age, high school GPA and academic self-efficacy were entered first, explaining $14 \%$ of the variance in the first semester GPAs. Language proficiency indicators were then added via stepwise regression, and the total variance explained by the whole model was $20 \%$. Self-perceived Korean proficiency was found to be the only significant predictor among language proficiency indicators, accounting for an additional $6 \%$.

As presented in Table 3, there were no significant predictors in the regression with undergraduate participants' first semester credit hours as the dependent variable. None of the language proficiency indicators entered into the regression via Step 2.

The results of graduate participants' multiple regression with first semester GPAs as the dependent variable are shown in Table 4. Gender, age, undergraduate GPA, and academic self-efficacy were entered first, explaining $16 \%$ of the variance in the first semester GPAs. Language proficiency indictors were then added via stepwise regression, and the total variance explained by the whole model was $38 \%$. In the final step, the significant predictors were undergraduate GPA $(\beta=.47, p=.001)$, academic self-efficacy $(\beta=-.32, p<.05)$, the TOPIK total score $(\beta=.48, p<.001)$, and the length of previous English study $(\beta=.25$, $p<.05)$. In Step 2 , with the presence of academic self-efficacy, the TOPIK total score accounted for an additional $18 \%$ of the variance in first semester GPAs, and the length of previous English study added another $4 \%$ to the variance in Step 3.

Note that academic self-efficacy was not a significant predictor when it was first entered into the regression. In fact, academic self-efficacy was not significantly correlated with graduate first semester GPA. However, when the TOPIK total score was entered, it became a significant predictor with a negative $\beta$ value.

Table 2 Hierarchical regression analysis of undergraduate students' first semester GPA ( $n=102)$

\begin{tabular}{|c|c|c|c|c|c|c|}
\hline Step & Adjusted $R^{2}$ & F change $\left(d f_{1}, d f_{2}\right)$ & Variable & Final $\beta$ & $t$ & $p$ \\
\hline \multirow[t]{4}{*}{1} & .14 & $5.12^{* * *}(4,97)$ & Gender & .21 & 2.16 & .03 \\
\hline & & & Age & -.07 & -.72 & .47 \\
\hline & & & High school GPA & .14 & 1.50 & .14 \\
\hline & & & Academic self-efficacy & .11 & 1.18 & .24 \\
\hline 2 & .20 & $8.01^{* *}(1,96)$ & Self-perceived Korean Proficiency & .27 & 2.83 & .006 \\
\hline
\end{tabular}


Table 3 Hierarchical regression analysis of undergraduate students' first semester credit hours $(n=102)$

\begin{tabular}{|c|c|c|c|c|c|c|}
\hline Step & Adjusted $R^{2}$ & $\mathrm{~F}$ change $\left(\mathrm{df}_{1}, \mathrm{df}_{2}\right)$ & Variable & Final $\beta$ & $t$ & $p$ \\
\hline \multirow[t]{4}{*}{1} & .02 & $1.61(4,97)$ & Gender & -.13 & -1.19 & .24 \\
\hline & & & Age & .19 & 1.79 & .08 \\
\hline & & & High school GPA & .02 & .19 & .85 \\
\hline & & & Academic self-efficacy & .01 & .11 & .91 \\
\hline
\end{tabular}

Academic self-efficacy may serve as a suppressor variable (see Cohen \& Cohen, 1983). Since academic self-efficacy was not significantly correlated with graduate first semester GPA, it was actually a typical or classic suppressor which invites the interaction with suppression. Suppression is present when either one of two independent variables' correlation with the dependent variable is less than the correlations between the two independent variables (Cohen \& Cohen, 1983). The correlation between the TOPIK total score and graduate first semester GPA was .40. Academic self-efficacy and graduate first semester GPA were not significantly correlated ( $r=.07$, n.s.), both of which were smaller than the correlation between the TOPIK total score and academic self-efficacy $(r=.43)$.

When suppression happens, it is common for one of the coefficients to become negative (academic self-efficacy in this case). The suppressor variable suppresses the unwanted variance in the independent variable with which it interacts, and enhances the relationship between that independent variable and dependent variable (Cohen \& Cohen, 1983). In this case, the $\beta$ value of the TOPIK total score has been enlarged by academic self-efficacy. In order to see the real contribution of the TOPIK total score, the regression with graduate first semester GPAs as the dependent variable was performed again without academic self-efficacy. The results are presented in Table 5.

Comparing Table 5 to Table 4, gender, age, and undergraduate GPA still accounted for $16 \%$ of the variance in the graduate first semester GPAs without academic self-efficacy. The TOPIK total score accounted for an additional $11 \%$ of the variance in graduate first semester GPAs, and the length of previous English study added another $6 \%$. The total variance explained was reduced from $38 \%$ to $33 \%$. Both the $\beta$ values of undergraduate GPA $(\beta=.34, p=.01)$ and the TOPIK total score $(\beta=.35, p<.01)$ were reduced while the $\beta$ value of the length of previous English study $(\beta=.28, p<.05)$ was increased. Note that the TOPIK total score still indicated a slightly higher $\beta$ value than undergraduate GPA.

Overall, it appears that academic self-efficacy did not play an influential role for the students investigated in the present study. In comparison, the mean correlation of academic self-efficacy and university GPA in Richardson et al.'s (2012) meta-analysis was

Table 4 Hierarchical regression analysis of graduate students' first semester GPA $(n=51)$

\begin{tabular}{|c|c|c|c|c|c|c|}
\hline Step & Adjusted $R^{2}$ & $F$ change $\left(\mathrm{df}_{1}, \mathrm{df}_{2}\right)$ & Variable & Final $\beta$ & $t$ & $p$ \\
\hline \multirow[t]{4}{*}{1} & .16 & $3.31^{*}(4,46)$ & Gender & .16 & 1.38 & .17 \\
\hline & & & Age & .02 & .14 & .89 \\
\hline & & & Undergraduate GPA & .47 & 3.54 & .001 \\
\hline & & & Academic self-efficacy & -.32 & -2.30 & .03 \\
\hline 2 & .34 & $13.84^{* * *}(1,45)$ & TOPIK total & .48 & 3.78 & .000 \\
\hline 3 & .38 & $4.19^{*}(1,44)$ & Length of previous English study & .25 & 2.05 & .047 \\
\hline
\end{tabular}


Table 5 Hierarchical regression analysis of graduate students' first semester GPA without academic self-efficacy $(n=51)$

\begin{tabular}{|c|c|c|c|c|c|c|}
\hline Step & Adjusted $R^{2}$ & $F$ change $\left(\mathrm{df}_{1}, \mathrm{df}_{2}\right)$ & Variable & Final $\beta$ & $t$ & $p$ \\
\hline \multirow[t]{3}{*}{1} & .16 & $4.24^{* *}(3,47)$ & Gender & .18 & 1.51 & .14 \\
\hline & & & Age & .01 & .09 & .93 \\
\hline & & & Undergraduate GPA & .34 & 2.71 & .01 \\
\hline 2 & .27 & $8.10^{* *}(1,46)$ & TOPIK total & .35 & 2.96 & .005 \\
\hline 3 & .33 & $4.57 *(1,45)$ & Length of previous English study & .28 & 2.14 & .04 \\
\hline
\end{tabular}

moderate $(r=.31)$, and in Robbins et al.'s (2004) meta-analysis, academic self-efficacy $(r=.38)$ was identified as the best predictor of university GPA and even had a higher $\beta$ coefficient than high school GPA in the regression.

With the multiple regression of graduate first semester credit hours as the dependent variable, Step 1 was not significant and the adjusted $\mathrm{R}$ square was -.07 , indicating that gender, age, undergraduate GPA, and academic self-efficacy were not significant predictors of graduate participants' first semester credit hours earned. Therefore, all four variables were excluded and the regression was performed again with only language proficiency indicators via stepwise regression. The results are shown in Table 6.

As indicated in Table 6, the TOPIK total score was entered into the model first, explaining $6 \%$ of the variance in graduate participants' first semester credit hours. Then the length of previous Korean study was entered, adding another $9 \%$ to the variance explained. In the final model, the TOPIK total score $(\beta=-.33, p<.05)$, the length of previous Korean study $(\beta=.42, p<.01)$, and self-perceived Korean proficiency $(\beta=-.30, p<.05)$ explained a total of $22 \%$ of the variance, which indicates that self-perceived Korean proficiency added an extra $7 \%$ to the explained variance.

\section{Discussion}

\section{Predictors of GPA and credit hours}

Based on the regression analyses, undergraduate GPA was predicted by gender and self-perceived Korean proficiency. In Richardson et al.'s (2012) comprehensive metaanalysis, the effect size estimates of gender $(r=.09)$ and age $(r=.08)$ were small. However, a much larger association with gender $(r=.32)$ was found in the present study indicating that, in the case of Chinese students studying at Korean universities, female undergraduate students obtained higher GPAs than males.

In Wait and Gressel's (2009) study, set in the context of an American accredited university located in the United Arab Emirates, gender was also found to be a significant predictor of GPA, and again female students earned higher overall GPAs than males. Wait and Gressel's (2009) further noted that an increasing TOEFL score had a greater

Table 6 Hierarchical regression analysis of graduate students' first semester credit hours $(n=51)$

\begin{tabular}{llllccc}
\hline Step & Adjusted $\mathrm{R}^{2}$ & $\mathrm{~F}$ change $\left(\mathrm{df}_{1}, \mathrm{df}_{2}\right)$ & Variable & Final $\beta$ & $t$ & $p$ \\
\hline 1 & .06 & $4.43^{*}(1,49)$ & TOPIK total & -.33 & -2.59 & .013 \\
2 & .15 & $6.09^{*}(1,48)$ & Length of previous Korean study & .42 & 3.12 & .003 \\
3 & .22 & $5.04^{*}(1,47)$ & Self-perceived Korean proficiency & -.30 & -2.24 & .03 \\
\hline Note: ${ }^{*} p<.05$ & & & & &
\end{tabular}


positive effect on GPA for female students than for males, which they claimed could be attributed to different learning style preferences between the genders. Other empirical studies in language testing, however, have not supported this finding.

In the present study, female undergraduate students showed higher high school GPAs, TOPIK scores, and ratings of self-perceived Korean and English proficiency and academic self-efficacy. These superior previous academic performances and perceptions could have contributed to their higher GPA in their undergraduate education.

There were no significant predictors of undergraduate credit hours earned. This finding was not surprising because among all the independent variables, only age indicated a small correlation with undergraduate GPA $(r=.22)$. However, in previous studies, language proficiency was found to have associations with undergraduate credit hours. For example, Stoynoff (1997) found a significant correlation between TOEFL scores and credits earned $(r=.23, p=.05)$ among the 77 international students who enrolled as freshmen at a university in the northwest of the United States. Johnson (1988) selected 27 undergraduate students at the University of Wisconsin-Green Bay in the United States with TOEFL scores ranging from 450 to 525 , and found a high correlation between TOEFL scores and credit hours earned $(r=.80, p<.01)$. The undergraduate participants investigated in the present study might have achieved adequate levels of language proficiency at the time of the study, or prior to their entrance into university, so that language proficiency no longer influenced the credit hours they registered for.

Graduate GPA was predicted by undergraduate GPA, academic self-efficacy, the TOPIK total score, and the length of previous English study. In the present study, undergraduate GPA $(r=.39)$ had a medium correlation and was the only significant predictor of graduate GPA in the initial regression model among all four traditional and psychological factors. This result is consistent with Wimberley et al.'s (1992) study that included international students from 27 different U.S. universities. They found that undergraduate GPA was the strongest predictor of graduate GPA. The present study confirmed that prior academic performance was indeed a strong predictor of GPA. Academic self-efficacy, however, was not a significant predictor when it was first entered into the regression and was determined to be a suppressor rather than a predictor.

Graduate credit hours earned were predicted by all three Korean proficiency indicators: the TOPIK total score, self-perceived Korean proficiency, and the length of previous Korean study. The former two predictors had negative $\beta$ coefficients. This is not unexpected as graduate students with poor Korean proficiency were required to take more credit hours to increase their Korean language skills. The positive $\beta$ coefficient of the length of previous Korean study actually implied that the longer graduate students studied Korean, the more credit hours they registered for, and the poorer their Korean proficiency. The length of previous Korean study was not correlated with the TOPIK total score or self-perceived Korean proficiency, indicating that more years of Korean study did not guarantee higher levels of Korean proficiency with this group of Chinese students. The length of previous Korean study represented the time from when students started their Korean courses until the time they participated in this study.

It was expected that graduate credit hours earned would exhibit less variation than undergraduate credit hours earned. This is because most graduate students in Korean universities register for 9 credit hours (3 courses) each semester (or at most 12 credit hours, 4 courses). However, the data in this study showed variation in graduate credit 
hours likely because graduate students with poor Korean proficiency were required to take Korean language courses along with their major courses. Given the link between increased credit hours and lower language proficiency, GPA remains to be and is a better indicator of academic success in the context of Chinese students studying in Korean universities. This finding contradicts previous research but illustrates the importance of context in exploring seemingly similar outcome variables (Graham, 1987; Johnson, 1988; Light et al., 1987; Stoynoff, 1997).

\section{Contribution of language proficiency}

Based on the findings of correlation analyses, the present study found that Korean language proficiency, as defined by test scores, had a moderate relationship with GPA even though previous research had mixed results regarding the relationship between TOEFL/IELTS and GPA. Furthermore, both the TOPIK total score and all subsection scores showed small to medium correlations to undergraduate GPA. TOPIK total, Vocabulary \& Grammar, and Writing showed medium correlations with graduate GPA. TOPIK Listening and Reading were not correlated with graduate GPA, likely because the scores on those two sections had ceiling effects.

Overall, TOPIK showed a small to medium correlation with GPA. In the regression model with graduate GPA as the dependent variable, the TOPIK total score was a significant predictor, and even had a slightly higher $\beta$ value than undergraduate GPA, the traditional significant predictor. The predictive validity of TOPIK, therefore, is found to be relatively strong compared with previous studies using English proficiency scores, and could serve well as one of the admission requirements to Korean universities.

Self-perceived Korean proficiency showed a stronger correlation with undergraduate GPA than the TOPIK total score and became the only predictor among language proficiency indicators. Also note that self-perceived Korean proficiency and the TOPIK total score had a medium correlation $(r=.45)$ among undergraduate participants. Even though the TOPIK total score showed a medium correlation with undergraduate GPA, it was not a significant predictor in the regression model. This finding suggests that correlation analyses alone might not be sufficient, and both correlation and regression analyses should be adopted in future prediction studies. This result is consistent with Xu's (1991) study, in which self-rated English proficiency was the major predictor while TOEFL was not significantly associated with the perceived level of academic difficulty. Also in Li et al.'s (2010) study of 178 international students in a university in England, self-perceived English writing ability instead of English test scores, was determined to be a significant predictor of academic success. This finding suggests that self-perceived language proficiency can predict academic success better than standardized language tests, and hence is also a strong (and sometimes better) indicator of language proficiency. In contrast, this study found that self-perceived English proficiency had a small correlation and was not a significant predictor of undergraduate GPA, indicating that English as a third language has limited influence on Chinese undergraduate GPAs in Korean universities.

All three Korean proficiency indicators, including the TOPIK total score, self-perceived Korean proficiency and the length of previous Korean study, were significant predictors of graduate credit hours earned. This finding indicates that each factor captured different 
aspects of language proficiency, and researchers should keep in mind that the different language proficiency indicators might be best employed together when exploring the relationships between language proficiency and academic success.

\section{Comparisons of undergraduate and graduate prediction models}

Since there were no significant predictors in the regression with undergraduate credit hours as the dependent variable, the results of regression with undergraduate and graduate GPA as dependent variables were compared. The regression analyses determined that the prediction patterns for undergraduate and graduate GPA were different, which is not surprising given the differing natures of undergraduate and graduate studies.

Gender was an effective predictor of undergraduate GPA, but not graduate GPA, and female undergraduates obtained higher GPAs than males. Compared to previous research, gender here indicated a stronger correlation with GPA among undergraduate participants, which may be due to the superior previous performance in both academics and language proficiency by female undergraduates. Certainly, additional research is needed to investigate the reasons behind this difference. Age was not a relevant factor in the present study, largely because Chinese students involved in both undergraduate and graduate studies in Korean universities generally belonged to the same age group.

For undergraduate students, prior academic performance and English proficiency did not have an effect on their academic success. Prior academic performance (high school GPA) was not a significant predictor probably due to the poor measurement quality of this variable, the high school graduation unified exam. The unified exam reports students' achievements on only four levels - excellent, good, moderate, and pass, likely an ordinal outcome. Amongst the 102 undergraduate students in the regression analyses, 66 (64.7\%) reported scores of "excellent" and "good." High schools in China do not have a GPA system to record class marks, making the National Matriculation Test the best representative of high school GPA. However, not all Chinese students who pursue undergraduate studies in Korea have taken this test. Therefore, the high school graduation unified exam was chosen to measure high school GPA in this study. For the reasons mentioned above, researchers should be cautious when using the high school graduation unified exam in future studies.

English proficiency was found to be a significant predictor of graduate GPA, but not undergraduate GPA, which might be attributed to the different characteristics of undergraduate and graduate studies. Undergraduate studies mainly focus on learning basic concepts or skills and lay foundations for life, work or future studies while graduate studies concentrate on more advanced topics and require deeper exploration of complex ideas. Graduate students in Korean universities need to read literature in English, the language in which most advanced research is published. In addition, English textbooks are often adopted in graduate courses in Korean universities. Hence it is not surprising that English proficiency would contribute to graduate students' academic success in Korean universities.

Academic self-efficacy was determined to be more of a suppressor than a predictor of graduate GPA. Although it did not directly contribute to graduate GPA, it did have an interaction with Korean proficiency. It is interesting that academic self-efficacy had no such interaction with undergraduate students' Korean proficiency. Language proficiency played an important role in academic success in the present study. It has been suggested that it is at lower levels of proficiency where language becomes an important predictor of academic success (Elder, 1993; Graham, 1987). Johnson (1988) claimed that with higher 
language proficiency (when language proficiency stops playing an important role in academic success), other factors such as motivation and academic aptitude/experience may become more important in academic success (also see Stoynoff, 1997). This might explain why academic self-efficacy was not very influential in the present study.

Note that graduate students indicated higher Korean proficiency than undergraduate students in this study. A series of independent $t$ tests showed that, compared to undergraduate participants, graduate participants' performance on all five language proficiency indicators were significantly superior. According to Elder (1993), above a certain level of language proficiency, many other factors - including scholastic aptitude, motivation, and interactive styles - are likely to interact with language ability. Hence, it is possible that the level of graduate students' Korean proficiency was largely above this level.

\section{Conclusion and implications}

This study investigated the key determinants of Chinese students' academic success in Korean universities, including gender, age, prior academic performance, academic selfefficacy, and especially Korean and English language proficiency including the TOPIK scores, self-perceived Korean and English proficiency, and the length of previous Korean and English study. Consistent with previous research, traditional factors including gender and prior academic performance were found to be effective predictors of academic success. However, academic self-efficacy did not play an influential role for Chinese students studying in Korean universities, possibly due to their levels of language proficiency.

Language proficiency had a moderate effect on Chinese students' academic success, which is consistent with previous studies that reported a positive statistically significant relationship between language proficiency and academic success. In this context particularly, Korean proficiency contributed to undergraduate GPA and graduate credit hours earned, whereas both Korean and English proficiency contributed to graduate GPA.

The different natures of undergraduate and graduate studies determined that the predictors of undergraduate and graduate students' academic success were different. The predictive validity of TOPIK is strong compared with previous prediction studies, indicating that TOPIK measures the Korean proficiency needed in academic settings and that TOPIK Intermediate (Levels $3 \& 4$ ) is the proper thresholds for international students who want to pursue further study in Korean universities. Furthermore, self-perceived language proficiency and the length of previous language study are effective alternative measures of language proficiency.

These findings have theoretical implications for future research. GPA is a stronger indicator of academic success than credit hours earned; hence GPA, instead of credit hours, might be used as a better indicator of academic success in future research. Standardized test score, self-perceived language proficiency, and the length of previous language study are all effective indicators of language proficiency, and these various indicators might be best employed together when investigating the relationships between language proficiency and academic success. It should be noted that only Chinese students were investigated in the present study and hence the findings may not be generalized to international students from other countries. 
The findings of this study also have practical implications. The TOPIK indicated a relatively strong predictive validity, and hence could serve well as an admission criterion. In addition, self-perceived language proficiency and the length of previous language study could also be used to complement the TOPIK score in admission decisions. As for graduate students, it may be helpful for the admission committee to include proof of English language proficiency as one of the entrance criteria. Language proficiency plays an important role in academic success, which is positive to both Korean universities and Chinese students who want to pursue further study in Korean universities. Universities can screen applicants and require intervention based on language proficiency, while students should be aware that they need to attain a certain level of language proficiency in order to achieve academic success.

The determinants of international students' academic success are complex and not yet completely understood, with language proficiency being only one of the factors contributing to international students' academic success. This study addressed the research gap by integrating theoretical constructs from both psychology and education, and also by exploring the relationships between language proficiency and academic success in a less researched test, TOPIK, and in two second languages, Korean and English, at the same time. The findings contribute to the overall understanding of international students' academic success, in particular the success of Chinese students studying in Korean universities. However, this study was also limited by the range restrictions of the sample and the limited factors investigated. These limitations should be kept in mind when interpreting the findings of this study.

Competing interests

The authors declare that they have no competing interests.

Authors' contributions

Both authors read and approved the final manuscript.

Received: 11 April 2015 Accepted: 29 May 2015

Published online: 01 July 2015

References

Ayers, JB, \& Quattlebaum, RF. (1992). TOEFL performance and success in a masters program in engineering. Educational and Psychological Measurement, 52(4), 973-975.

Cho, Y, \& Bridgeman, B. (2012). Relationship of TOEFL iBT scores to academic performance: Some evidence from American universities. Language Testing, 29(3), 421-442.

Cohen, J, \& Cohen, P. (1983). Applied multiple regression/correlation analysis for the behavioral sciences (2nd ed.). Hillsdale, NJ: Lawrence Erlbaum Associates, Inc., Publishers.

Cotton, F, \& Conrow, F. (1998). An investigation of the predictive validity of IELTS amongst a group of international students studying at the University of Tasmania. IELTS Research Reports, 1, 72-115.

Criper, C, \& Davies, A. (1988). ELTS research report 1(i): ELTS validation project report. Cambridge: The British Council/ University of Cambridge Local Examinations Syndicate

Dooey, P, \& Oliver, R. (2002). An investigation into the predictive validity of the IELTS Test as an indicator of future academic success. Prospect, 17(1), 36-54.

Duncan, TG, \& McKeachie, WJ. (2005). The making of the motivated strategies for learning questionnaire. Educational Psychologist, 40(2), 117-128.

Elder, C. (1993). Language proficiency as a predictor of performance in teacher education. Melbourne Papers in Language Testing, 2(1), 72-95.

Fabrigar, LR, Wegener, DT, MacCallum, RC, \& Strahan, EJ. (1999). Evaluating the use of exploratory factor analysis in psychological research. Psychological Methods, 4(3), 272-299.

Feast, V. (2002). The impact of IELTS scores on performance at university. International Education Journal, 3(4), $70-85$.

Gao, F. (2010). Learning Korean language in China: Motivations and strategies of non-Koreans. International Journal of Bilingual Education and Bilingualism, 13(3), 273-284.

Graham, JG. (1987). English language proficiency and the prediction of academic success. TESOL Quarterly, 21(3), 505-521.

Gukrip Gukje Gyoyukwon [National Institute for International Education]. (2010). TOPIK Hangukeo Neungnyeong Siheom [Test of Proficiency in Korean]. Retrieved February 23, 2013, from http://www.topik.go.kr/jsp/hp/sub/ subLocation.do?MENU_SEQ=20101040\&UP_MENU_SEQ=2010104. 
Gukrip Gukje Gyoyukwon [National Institute for International Education]. (2013). Study in Korea: run by Korean government. Retrieved March 9, 2013, from http:/www.studyinkorea.go.kr/en/sub/overseas_info/korea_edu/edu_system.do.

Heil, DK, \& Aleamoni, LM (1974). Assessment of proficiency in the use and understanding of English by foreign students as measured by the Test of English as a Foreign Language. Urbana: University of Illinois (ERIC Document Reproduction Service No. ED 093948).

Hill, K, Storch, N, \& Lynch, B. (1999). A comparison of IELTS and TOEFL as predictors of academic success. IELTS Research Reports, 2, 62-73.

Johnson, P. (1988). English language proficiency and academic performance of undergraduate international students. TESOL Quarterly, 22(1), 164-168.

Kerstjens, M, \& Nery, C. (2000). Predictive validity in the IELTS test: A study of the relationship between IELTS scores and students' subsequent academic performance. IELTS Research Reports, 3, 85-108.

Krausz, J, Schiff, A, Schiff, J, \& Van Hise, J. (2005). The impact of TOEFL scores on placement and performance of international students in the initial graduate accounting class. Accounting Education, 14(1), 103-111.

Lee, Y-J, \& Greene, J. (2007). The predictive validity of an ESL placement test: A mixed methods approach. Journal of Mixed Methods Research, 1(4), 366-389.

Li, G, Chen, W, \& Duanmu, J-L. (2010). Determinants of international students' academic performance: A comparison between Chinese and other international students. Journal of Studies in International Education, 14(4), 389-405.

Light, RL, \& Teh-Yuan, W. (1991). Soviet students at U.S. colleges: Social perceptions, language proficiency, and academic success. TESOL Quarterly, 25(1), 179-185.

Light, RL, Xu, M, \& Mossop, J. (1987). English proficiency and academic performance of international students. TESOL Quarterly, 21(2), 251-261.

McKenzie, K, \& Schweitzer, R. (2001). Who succeeds at university? Factors predicting academic performance in first year Australian university students. Higher Education Research \& Development, 20(1), 21-33.

Nelson, CV, Nelson, JS, \& Malone, BG. (2004). Predicting success of international graduate students in an American university. College and University, 80(1), 19-27.

Ockey, GJ. (2014). Exploratory factor analysis and structural equation modeling. In AJ Kunnan (Ed.), The companion of language assessment (pp. 1224-1244). NJ: John Wiley \& Sons. doi:10.1002/9781118411360.wbcla114.

Pintrich, PR. (2004). A conceptual framework for assessing motivation and self-regulated learning in college students. Educational Psychology Review, 16(4), 385-407.

Pintrich, PR, Smith, DAF, Garcia, T, \& McKeachie, WJ. (1991). A manual for the use of the Motivated Strategies for Learning Questionnaire (MSLQ). Ann Arbor: University of Michigan, National Center for Research to Improve Postsecondary Teaching and Learning.

Rao, N, Moely, BE, \& Sachs, J. (2000). Motivational beliefs, study strategies, and mathematics attainment in high- and low-achieving Chinese secondary school students. Contemporary Educational Psychology, 25(3), 287-316.

Rao, N, \& Sachs, J. (1999). Confirmatory factor analysis of the Chinese version of the motivated strategies for learning questionnaire. Educational and Psychological Measurement, 59(6), 1016-1029.

Richardson, M, Abraham, C, \& Bond, R. (2012). Psychological correlates of university students' academic performance: A systematic review and meta-analysis. Psychological Bulletin, 138(2), 353-387.

Robbins, SB, Lauver, K, Le, H, Davis, D, Langley, R, \& Carlstrom, A. (2004). Do psychosocial and study skill factors predict college outcomes? A meta-analysis. Psychological Bulletin, 130(2), 261-288.

Rosenthal, R, \& Rubin, DB. (1982). A simple, general purpose display of magnitude of experimental effect. Journal of Educational Psychology, 74(2), 166-169.

Sachs, J, Law, YK, Chan, CKK, \& Rao, N. (2001). A nonparametric item analysis of the motivated strategies for learning questionnaire - Chinese version. Psychologia, 44(3), 197-208.

Sackett, PR, Borneman, MJ, \& Connelly, BS. (2008). High-stakes testing in higher education and employment: Appraising the evidence for validity and fairness. American Psychologist, 63(4), 215-227.

Schrader, WB. (1965). A taxonomy of expectancy tables. Journal of Educational Measurement, 2(1), 29-35.

Shin, K-J. (2011). Maqujapi yuchineun dok, hangukeo neungnyeokbuteo geomjeunghaeya [The poison of indiscriminate selection, from the verification of the Korean Language Proficiency]. Joongang: SUNDAY. Retrieved February 23, 2013, from http://sunday.joins.com/article/view.asp?aid=23409.

Stoynoff, S. (1997). Factors associated with international students' academic achievement. Journal of Instructional Psychology, 24(1), 56-68.

Strenze, T. (2007). Intelligence and socioeconomic success: A meta-analytic review of longitudinal research. Intelligence, 35(5), 401-426.

Wait, IW, \& Gressel, JW. (2009). Relationship between TOEFL score and academic success for international engineering students. Journal of Engineering Education, 98(4), 389-398.

Wimberley, DW, McCloud, DG, \& Flinn, WL. (1992). Predicting success of Indonesian graduate students in the United States. Comparative Education Review, 36(4), 487-508.

Woodrow, L. (2006). Academic success of international postgraduate education students and the role of English proficiency. University of Sydney Papers in TESOL, 1, 51-70.

Wu, J, \& Cheng, B. (1992). Jilide xuexicelue liangbiaozhi xiuding [A revised version of the Motivated Strategies for Learning Questionnaire]. Ceyan Niankan, 39, 59-78.

Xu, M. (1991). The impact of English-language proficiency on international graduate students' perceived academic difficulty. Research in Higher Education, 32(5), 557-570.

Yu, G. (2013). Gyogwabu, "Hangukeo Neungnyeong Siheom (TOPIK) Nujeok Jiwonja 100manmyeony Dolpa" [Ministry of Education, Science and Technology, "The accumulated number of TOPIK applicants exceeded 1 million"] News Wave. Retrieved from http://www.newswave.kr/sub_read.html?uid=244151\&section. 